\title{
Layered Horizons: a Geospatial Humanities Research Platform
}

\author{
Andrew Burrell \\ andrew.burrell@uts.edu.au \\ University of Technology Sydney \\ Sydney, NSW, Australia
}

\author{
Rachel Hendery \\ r.hendery@westernsydney.edu.au \\ Western Sydney University \\ Sydney, NSW, Australia
}

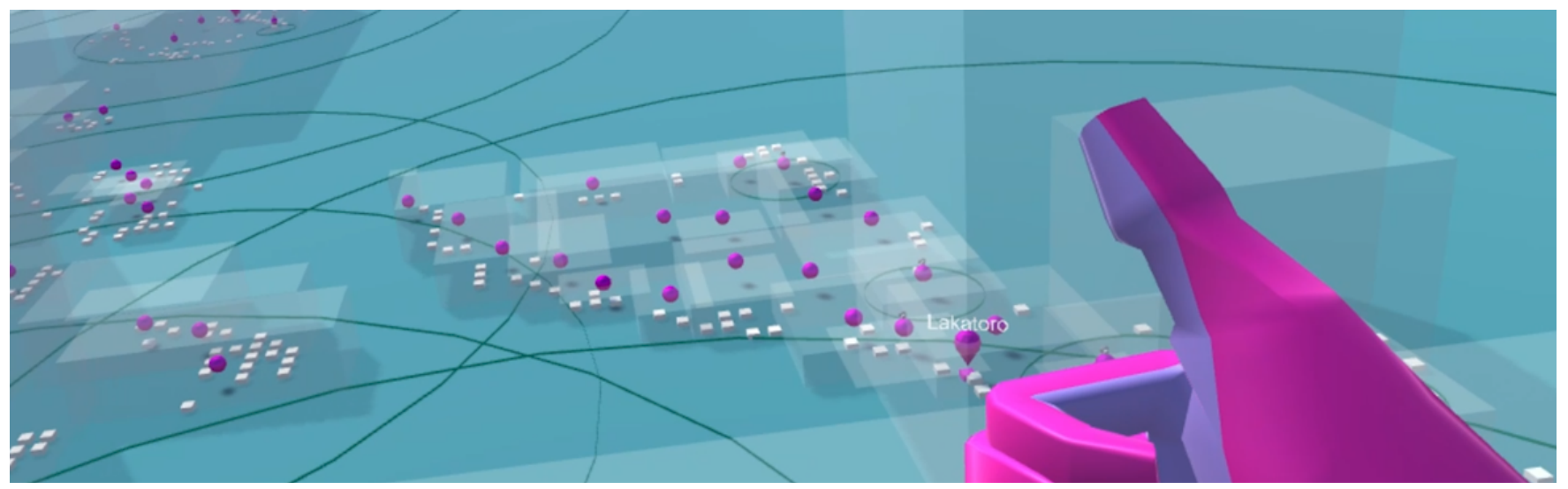

Figure 1: Thumbs up: a gesture-based interaction in Layered Horizons

\begin{abstract}
In this demo we showcase Layered Horizons, a Virtual Reality (VR) experience we have developed for use in an ARC-funded research project, Waves of Words: Mapping and Modelling Australia's Pacific Past. This platform allows users to connect different geospatial datasets (for our purposes, from the humanities and social sciences) into layers that can then be explored by the use of natural gesture and body movement. This kind of interaction design in VR takes full advantage of the media's affordances, without relying on metaphors from other interactive media, yet being familiar enough as to engender intuitive and meaningful use. We demonstrate how the platform is currently being used to connect linguistic data (word lists) with archaeological data (e.g. on the spread of bananas through the Asia-Pacific region, or canoe styles found in different locations) and anthropological data (e.g. shared cultural features like chieftainship systems or kinship systems). Taking into account what we also know about Pacific navigation and simulated canoe travel, we can therefore build a complex layered map of the region over time that allows us to better discover probable human migration and contact patterns.
\end{abstract}

\section{KEYWORDS}

interfaces, gesture, leap motion, virtual reality, languages, research platform, data visualisation, research through design

Permission to make digital or hard copies of part or all of this work for personal or classroom use is granted without fee provided that copies are not made or distributed for profit or commercial advantage and that copies bear this notice and the full citation on the first page. Copyrights for third-party components of this work must be honored For all other uses, contact the owner/author(s).

VRST '19, November 12-15, 2019, Parramatta, NSW, Australia

(c) 2019 Copyright held by the owner/author(s).

ACM ISBN 978-1-4503-7001-1/19/11.

https://doi.org/10.1145/3359996.3365052
ACM Reference Format:

Andrew Burrell and Rachel Hendery. 2019. Layered Horizons: a Geospatial Humanities Research Platform. In 25th ACM Symposium on Virtual Reality Software and Technology (VRST '19), November 12-15, 2019, Parramatta, NSW, Australia. ACM, New York, NY, USA, 2 pages. https://doi.org/10.1145/ 3359996.3365052

\section{OVERVIEW}

Layered Horizons is a Research through Design and Digital Humanities project that brings together disparate data sets from linguistics, anthropology, geography and archaeology-within virtual realityto create visualisations that investigate the extent and nature of ancient contact between First Peoples of Australia and the AsiaPacific region.

In the Layered Horizons VR project we bring together these data sets by spatialising them in a relatively abstract three-dimensional map, or datascape, of the Asia-Pacific, and allowing the user to move through the region and interrogate data points with natural gesture. The experience is designed in Unity and uses an HTC Vive headset equipped with a LeapMotion device for hand tracking. Data for this version of the demo is drawn from a number of sources, including the PARADISEC [Thieberger and Barwick 2012] and OLAC databases [Bird and Simons 2001], team members' own research data, as well as published research papers [e.g. Perrier et al. 2011].

Semi-transparent volumes, as shown in Figure 1 are used to connect regions of the map, to represent relationships between islands or languages, or the extent of a feature or a language. Where appropriate, the datapoints are associated with audio snippets. The geographical space can be warped around the user to represent relationships between places, e.g. bringing islands closer together if the data suggest they have a closer relationship, and further apart if not. 


\section{NOVELTY}

Virtual Reality has not yet been widely adopted in humanities and social sciences research, and even less so for the purposes of humanities and social sciences data visualisation. The VR experiences in these disciplines tend more often to be used for teaching [e.g. Brick et al. 2019], or outreach and engagement [e.g. Trescak et al. 2016], or for the burgeoning area of cultural heritage preservation [Bekele et al. 2018].

Unlike the more public-facing and applied goals of such projects, Layered Horizons is primarily a research tool. It facilitates discovery of relationships within and between humanities and/or social sciences datasets, and illuminates geographical locations that are likely to be significant for future research.

\section{USEFULNESS}

Layered Horizons is currently already being used by a team of researchers for an ARC Discovery Project in order to link together the very different kinds of data that the various team members are working with. We have been able to use it to identify subregions of the Pacific that are most closely connected, culturally, linguistically, and geographically in terms of canoe travel logistics. We expect to be able to use it further to discover relationships between datasets, as well as outlier datapoints in these datasets that are in need of further investigation and explanation.

As the VR project is intended as a research tool, we have designed the experience without locking its affordances to specific kinds of data or use cases, so that a researcher can choose what kinds of data points to display, what to represent with the transparent volumes, and what to base the warping of space on, for example.

All data are imported from csv files, allowing it to be used by humanities researchers without a strong technical background.

\section{APPLICATIONS}

The design framework and technical platform developing out of Layered Horizons is proving invaluable in creating new VR experiences, not only for research itself, but for the dissemination of research and other information, and has fed into a number of related projects. As we make the code and project frameworks publicly available under a permissive open source licence, we believe they will be valuable to other research projects in the future with geospatial data visualisation needs.

\section{INTERACTION}

In this demo, users will put on an HTC Vive headset, equipped with a LeapMotion camera, and will use natural gestures and body movement to explore the Vanuatu region, viewing datapoints from linguistic, anthropological and archaeological datasets. The user can move up, down, and through the region, viewing the datascape from different perspectives, and interacting with individual datapoints to learn more information. Datapoints (represented as white boxes or pink balls in Figure 1) can be touched or scooped up to trigger a display of associated metadata. By moving near data that has an audio component (e.g. language information), audio is triggered. Movement is controlled by pointing with a finger or a flat hand (for different speeds of movement).

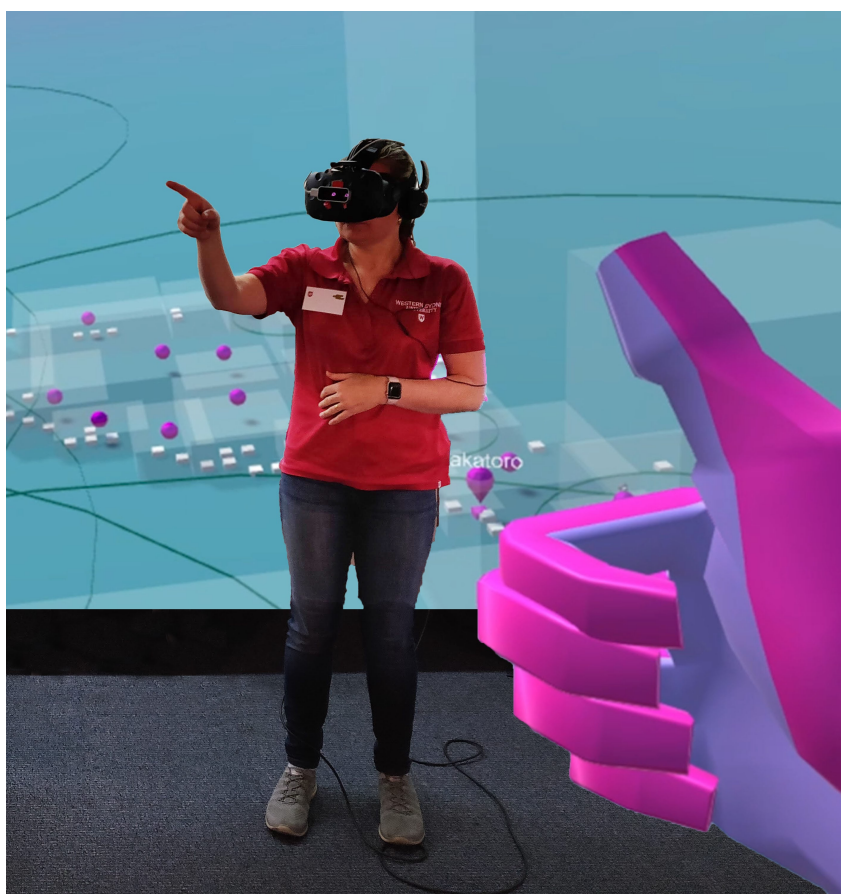

Figure 2: Composited image by Andrew Burrell, of users interacting with Layered Horizons.

\section{REFERENCES}

Mafkereseb Kassahun Bekele, Roberto Pierdicca, Emanuele Frontoni, Eva Savina Malinverni, and James Gain. 2018. A survey of augmented, virtual, and mixed reality for cultural heritage. Fournal on Computing and Cultural Heritage ( $(\mathrm{OOCCH}) 11,2$ (2018), 7

Steven Bird and Gary Simons. 2001. The OLAC metadata set and controlled vocabularies. In Proceedings of the ACL 2001 Workshop on Sharing Tools and Resources-Volume 15. Association for Computational Linguistics, 7-18.

Billy Brick, Tiziana Cervi-Wilson, Sean Graham, Tsvetan Tsankov, Michael Loizou, Nina Godson, and Kelly Ryan. 2019. 1Multilingual immersive communication technology: repurposing virtual reality for Italian teaching. In New educational landscapes: innovative perspectives in language learning and technology. Researchpublishing. net, 5-10.

Xavier Perrier, Edmond De Langhe, Mark Donohue, Carol Lentfer, Luc Vrydaghs, Frédéric Bakry, Françoise Carreel, Isabelle Hippolyte, Jean-Pierre Horry, Christophe Jenny, et al. 2011. Multidisciplinary perspectives on banana (Musa spp.) domestication. Proceedings of the National Academy of Sciences 108, 28 (2011), 11311-11318.

Nicholas Thieberger and Linda Barwick. 2012. Keeping records of language diversity in Melanesia: The Pacific and Regional Archive for Digital Sources in Endangered Cultures (PARADISEC). Melanesian languages on the edge of Asia: Challenges for the 21st Century (2012), 239-53.

Tomas Trescak, Anton Bogdanovych, Simeon Simoff, Melissa Williams, and Terry Sloan. 2016. Virtual dreaming: simulating everyday life of the Darug people. In International Conference on Intelligent Virtual Agents. Springer, 509-512. 